\title{
Familial amyloidotic polyneuropathy with severe renal involvement in association with transthyretin Gly47Glu in Dutch, British and American-Finnish families
}

\author{
Elizabeth B. Haagsma ${ }^{1}$, Philip N. Hawkins ${ }^{2}$, Merrill D. Benson ${ }^{3}$, Helen J. Lachmann ${ }^{2}$, \\ Alison Bybee ${ }^{2}$ and Bouke P.C. Hazenberg ${ }^{4}$
}

1. Department of Gastroenterology and Hepatology, University Hospital, Groningen, The Netherlands

2. National Amyloidosis Centre, Royal Free Hospital, Royal Free and University College Medical School, London, UK

3. Department of Pathology and Laboratory Medicine, Indiana University School of Medicine, Indianapolis, USA

4. Department of Rheumatology, University Hospital, Groningen, The Netherlands

KEY WORDS: transthyretin, inherited amyloidosis, Gly47Glu, renal failure

ABbreVIATIONS: FAP=familial amyloidotic polyneuropathy; TTR=transthyretin; Gly=glycine; Glu=glutamic acid; Val=valine; Met =methionine $; \quad G F R=$ glomerular filtration rate; $S A P=$ Serum amyloid $P$ component $; \quad$ DPD $=3,3-$ diphosphono-1,2propanodicarboxylic acid

\begin{abstract}
Familial amyloidotic polyneuropathy (FAP) is an autosomal dominant disorder associated with more than 80 different transthyretin (TTR) mutations. The clinical features of FAP are broad and variable, but knowledge of the pattern and natural history of disease associated with particular mutations nevertheless offers the best guidance for management of individual patients, including the role and timing of treatment by orthotopic liver transplantation. FAP in association with TTR Gly47Glu has been described previously in an Italian kindred, and we report here its phenotype in 7 additional patients from Dutch, British, and American (Finnish) families. Characteristic clinical features included amyloid cardiomyopathy and autonomic failure but, unusually, moderate to severe renal failure was present in more than half of the cases. Only four patients were deemed to be sufficiently fit to undergo orthotopic liver transplantation, and clinical
\end{abstract}

deterioration was generally rapid. These observations support early intervention with orthotopic liver transplantation in patients with FAP associated with TTR Gly47Glu.

\section{Introduction}

$\mathrm{F}$ amilial amyloidotic polyneuropathy (FAP) is an autosomal dominant disorder associated with more than 80 different transthyretin (TTR) mutations $^{1}$. The disease is most commonly associated with the TTR Val30Met variant, and most other pathogenic mutations are very rare. FAP characteristically presents with progressive peripheral and autonomic neuropathy, but cardiac amyloid, which is often sub-clinical at diagnosis, is frequent and may dominate the clinical picture, especially in some non Met30 mutations ${ }^{2}$. Circulating TTR is almost entirely produced in the liver

Correspondence: Dr. Elizabeth B. Haagsma, Department of Gastroenterology and Hepatology, University Hospital Groningen, Hanzeplein 1, P.O. Box 30.001, 9700 RB Groningen, The Netherlands

Tel: 0031-50-3616161_Fax: 0031-50-3613151_E-mail: e.b.haagsma@int.azg.nl 
Amyloid: J. Protein Folding Disord. 11, 44-49 (2004)

and orthotopic liver transplantation is now used widely in a bid to halt disease progress, since it substantially removes variant TTR from the plasma. Unfortunately, amyloid cardiomyopathy may progress despite liver transplantation, presumably reflecting the propensity for normal wild-type TTR to be deposited as amyloid in the heart ${ }^{3,4}$. Although amyloid is deposited in the kidneys in many patients with FAP, clinically significant renal impairment is very rarely part of the natural history of this disease. Although the correlation between genotype and phenotype in FAP is very inconsistent, knowledge of the pattern and natural history of disease associated with particular mutations nevertheless offers the best guidance for management of individual patients, including the role and timing of treatment by orthotopic liver transplantation. FAP in association with TTR Gly47Glu has been identified previously ${ }^{5}$, and its clinical features have been described in an Italian kindred ${ }^{6}$. We report here its broader phenotype, notably including frequent severe renal disease, in members of Dutch, British and American families.

\section{Patients}

An overview and additional details of the following 7 cases are listed in Table 1. Figure 1 shows the pedigree of the Dutch family.

The propositus, Case A III-3, born in the southern Netherlands with distant forbears in Germany, was a healthy man until the age of 53 when he developed nausea and weight loss, followed by diarrhea and impotence. Within one year he had paresthesiae in his hands and feet. The diagnosis of FAP was established in 2000 when he was 55 years old, at which time evaluation with a view to possible liver transplantation confirmed mild polyneuropathy, moderate bladder dysfunction and cardiomyopathy, but no vitreous opacities. Additional findings were advanced renal insufficiency (GFR $15 \mathrm{ml} / \mathrm{min}$ ), with biopsy confirmation of renal amyloid deposits. Combined kidney-liver transplantation was ultimately precluded by ischaemic heart disease.

Concerning the family: his mother (Case A II-4) died at the age of 72 with end-stage amyloidosis. The diagnosis of amyloid had been established one year earlier, in 1985. She had suffered from substantial wasting, diarrhoea, dependent skin ulcers, orthostasis, and severe renal insufficiency (creatinine clearance $10 \mathrm{ml} / \mathrm{min}$ ). Her sister (Case A II-1) died at age 62 . Clinical details are incomplete, but she had developed gastrointestinal symptoms at age 54, followed by autonomic and motor neuropathy, and cardiac symptoms. Extensive amyloid deposition was confirmed at autopsy in 1968. Her son (Case A III-1) died at age 42 in 1982 without a histological diagnosis, but developed orthostatic hypotension, walking difficulties, and gastrointestinal problems at age 35 years indicating that FAP was probable. The maternal mother (Case A I-1) died at age 94, and the maternal father (Case A I2) at age 50 of unknown causes.

Case A III-4 is one of four sisters of the propositus. At the age of 49 she underwent surgical release for carpal tunnel syndrome. She was subsequently confirmed to be heterozygous for TTR Gly47Glu after the diagnosis of FAP had been established in her brother, and by the age of 51 had developed symptoms of fatigue and mild polyneuropathy. Further evaluation demonstrated mild amyloid cardiomyopathy, moderate orthostasis, mildly impaired renal function (GFR $76 \mathrm{ml} / \mathrm{min}$ ), disturbed gastric emptying, slight bladder dysfunction, but no vitreous opacities. She was considered to be eligible for orthotopic liver transplantation, which was performed without major complications.

Case A III-5 is a sister, 51 years old without complaints. Evaluation however revealed an impaired renal function (GFR $68 \mathrm{ml} / \mathrm{min}$ ), no proteinuria, and slight bladder dysfunction. Amyloid deposits were found in subcutaneous fat and stomach mucosa. She is listed for liver transplantation.

Case A IV-1 is the daughter of the elder brother of the propositus, Case A III-2, who at age 64 denies any symptoms of FAP and does not want to be tested for the mutation. At the age of 33 years she required surgical release for carpal tunnel syndrome, and from age 40 years developed progressive weight loss, orthostatic hypotension and very painful paraesthesiae. Evaluation in 2002, at the age of 43, demonstrated severe amyloid cardiomyopathy and extensive autonomic dysfunction with symptomatic orthostasis and marked disturbance of gastrointestinal motility. She had a sensory polyneuropathy and modestly impaired renal function. Her symptoms responded well to nasogastric feeding, analgesia comprising clonazepam and gabapentin, and the introduction of fludrocortisone. In addition to TTR Gly47Glu, the nonamyloidogenic TTR variant His90Asn was present in this patient. She received a combined liver and heart transplantation in a centre elsewhere. The heart was severely affected by amyloid deposition. Her clinical condition remained poor afterwards with severe feeding problems and she developed end stage renal failure requiring haemodialysis. She died suddenly almost four months after transplantation.

Case B and his family come from east London, Great Britain. At the age of 44 he noticed ankle edema and progressive weight loss. He subsequently developed paraesthesiae, particularly affecting the hands, erectile impotence and mild postural hypotension. There were no complaints of bowel or bladder 
Table 1. Patient characteristics of the ATTR Gly47Glu FAP families

\begin{tabular}{|c|c|c|c|c|c|c|c|}
\hline & Case A II-4 & Case A III-3 & Case A III-4 & Case A III-5 & Case A IV-1 & Case B & Case C \\
\hline Country & Netherlands & Netherlands & Netherlands & Netherlands & Netherlands & England & USA (Finland) \\
\hline Male/female & $\mathrm{F}$ & M & $\mathrm{F}$ & $\mathrm{F}$ & $\mathrm{F}$ & $M$ & M \\
\hline Age at first symptom & 69 & 53 & 49 & 51 & 33 & 44 & 35 \\
\hline Age at first evaluation & 71 & 55 & 51 & 51 & 43 & 46 & 37 \\
\hline Year of evaluation & 1985 & 2001 & 2001 & 2003 & 2002 & 2000 & 2002 \\
\hline First symptom & $\begin{array}{c}\text { nausea } \\
\text { weight loss }\end{array}$ & $\begin{array}{c}\text { nausea } \\
\text { weight loss }\end{array}$ & $\begin{array}{c}\text { fatigue } \\
\text { CTS }\end{array}$ & $\begin{array}{c}\text { bladder } \\
\text { dysfunction }\end{array}$ & CTS & edema & $\begin{array}{c}\text { erectile } \\
\text { impotence }\end{array}$ \\
\hline \multicolumn{8}{|l|}{ Polyneuropathy } \\
\hline Sensory & $?$ & + & $+1-$ & $+1-$ & + & + & + \\
\hline Motor & $?$ & + & - & - & - & + & + \\
\hline Autonomic & ++ & ++ & + & - & +++ & +++ & +++ \\
\hline \multicolumn{8}{|l|}{ Cardiac function } \\
\hline Amyloid present & $?$ & + & + & - & + & + & + \\
\hline Conduction defect & $?$ & ++ & + & - & - & + & - \\
\hline Systolic/diastolic dysfunction. & $?$ & + & - & - & ++ & ++ & ++ \\
\hline Septum thickness ( $\leq 11 \mathrm{~mm})$ & 'thickened' & $12 \mathrm{~mm}$ & $16 \mathrm{~mm}$ & $12 \mathrm{~mm}$ & $16 \mathrm{~mm}$ & $19 \mathrm{~mm}$ & $13 \mathrm{~mm}$ \\
\hline Left ventricle thickness $(\leq 11 \mathrm{~mm})$ & 'thickened' & $13 \mathrm{~mm}$ & $?$ & $12 \mathrm{~mm}$ & $14 \mathrm{~mm}$ & $20 \mathrm{~mm}$ & $13 \mathrm{~mm}$ \\
\hline Coronary disease & $?$ & ++ & - & - & - & $?$ & - \\
\hline \multicolumn{8}{|l|}{ Renal function } \\
\hline Creatinine clearance, $\mathrm{ml} / \mathrm{min}$ & 10 & 25 & 95 & 70 & 95 & 54 & $44^{*}$ \\
\hline GFR, ml/min & nt & 15 & 79 & 68 & $\mathrm{nt}$ & nt & $?$ \\
\hline Proteinuria, g/day & $?$ & 0.3 & 0.2 & 0.2 & 0.2 & 0.2 & 0.1 \\
\hline \multicolumn{8}{|l|}{ Bladder function } \\
\hline Infections & ++ & - & + & - & + & - & - \\
\hline Dysfunction ${ }^{\star *}$ & nt & ++ & + & + & - & - & - \\
\hline Self-catheterisation & - & - & - & - & - & - & - \\
\hline \multicolumn{8}{|l|}{ Gastrointestinal tract } \\
\hline Weight loss & ++ & ++ & - & - & +++ & + & +++ \\
\hline Disturbed motility & nt & + & + & - & ++ & - & +++ \\
\hline Vitreous opacities & - & - & - & - & - & - & - \\
\hline \multicolumn{8}{|l|}{ Amyloid deposits (pathology) } \\
\hline Heart & $\mathrm{nt}$ & nt & $\mathrm{nt}$ & $\mathrm{nt}$ & +++ & + & nt \\
\hline Small intestine & + & ++ & + & + & + & $\mathrm{nt}$ & nt \\
\hline Rectal mucosa & + & +++ & nt & nt & nt & nt & + \\
\hline
\end{tabular}

nt: denotes not tested; ?: denotes unknown; *denotes that the clearance was measured not before, but after liver transplantation

**bladder dysfunction as measured by urodynamic testing 


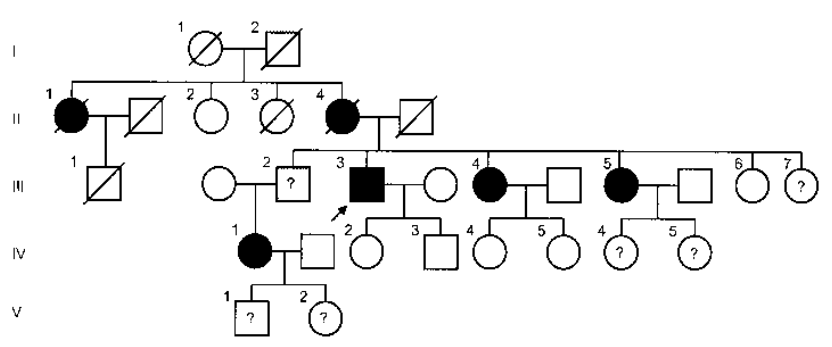

FIGURE 1: Pedigree of the Dutch family. Darkened square or circle means affected individual. A grey shaded square or circle means probable carrier of the mutation. The arrow denotes the propositus Case A III-3. The question mark denotes family members not tested for the mutation.

dysfunction. Further investigations in 2000 when he was aged 46 years, demonstrated renal impairment (creatinine clearance $54 \mathrm{ml} / \mathrm{min}$ ), and severe amyloid cardiomyopathy. No vitreous amyloid deposits were found. Orthotopic liver transplantation was not considered to be feasible due to his poor cardiac status. He died in 2002 following rapid and severe progression of sensory, motor and autonomic neuropathy, and progression of renal failure to virtual end stage disease. The TTR mutation in this patient was reported earlier ${ }^{7}$. With respect to the family, his father had died suddenly at the age of 67 and his mother is healthy at age 76. A 50 year old brother is without symptoms. Three children below 25 years of age are healthy.

Case C is a 39 year old Russian male physicist who immigrated to the United States and who developed erectile dysfunction at 35 and subsequently weight loss and diarrhea. He also developed mild peripheral neuropathy and increasing fatigue. At age 37 a diagnosis of amyloidosis was made by abdominal fat pad aspirate and subsequently confirmed by rectal biopsy. Shortly thereafter, at age 37, he had orthotopic liver transplantation. His course post-surgery was complicated by thrombosis of the hepatic artery ten days after surgery requiring retransplantation. After that time he was maintained on tacrolimus for immunosuppression. His subsequent course has been one of increasing autonomic dysfunction with severe hypotension, frequently causing difficulty with standing and walking. He was first evaluated at The Indiana University Amyloid Research Center in 2002 when he was 39 years of age. Echocardiogram was consistent with amyloid cardiomyopathy showing diastolic dysfunction and left ventricular wall and septum thickness of $13 \mathrm{~mm}$. The left atrial diameter was 3.9 centimeters. Technetium pyrophosphate scintigraphy showed marked uptake by the heart which was consistent

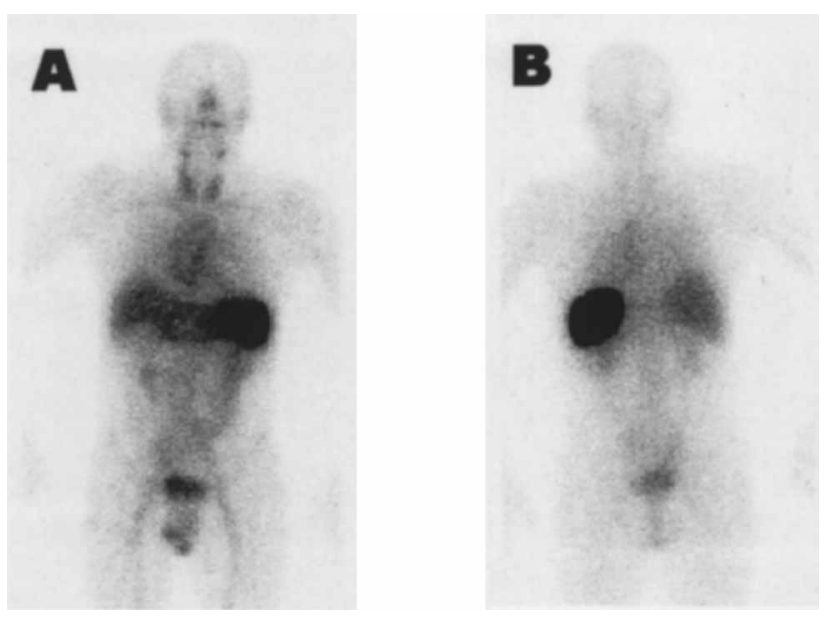

FIGURE 2: SAP scintigraph (24 hours after administration of SAP) of the propositus Case A III-3. Front view (A) and back view $(B)$ with intensive uptake of the spleen $(++)$ and slightly increased uptake of the kidneys $(+)$. Beside the increased uptake of the spleen and kidneys the expected normal picture of blood pool activity in heart and major blood vessels is visible as well as some radioactive degradation products in stomach and urinary bladder.

with amyloid infiltration and also uptake by the thyroid. The liver/spleen scan was within normal limits. Renal function was decreased with a creatinine clearance of $44 \mathrm{ml} / \mathrm{min}$. Following the evaluation the patient has continued to have severe orthostasis. The patient's father died at age 47 in Russia having had diarrhea and low blood pressure, but he did not have a post-mortem examination. The mother is 65 years old and alive. He has no siblings and his father was also an only child. The patient relocated from Uzbekistan and the paternal grandfather had been relocated from Finland to Uzbekistan during the reign of Stalin.

\section{SAP scintigraphy}

Highly purified human SAP was labelled with 123Iodine according to the method described by Mather and Ward ${ }^{8}$. A dose of $200 \mathrm{MBq}$ 123I-SAP containing $100 \mu \mathrm{g}$ protein was given as an intravenous bolus? . Thyroid uptake of free iodine was prevented by oral administration of potassium iodide. After $24 \mathrm{~h}$ anterior and posterior whole body scans were acquired using large-field-of-view rectangular gamma cameras. Increased uptake of tracer in organs was assessed visually ${ }^{10}$. Figure 2 shows the scintigraphs of one of the Dutch patients: increased uptake of the spleen $(++)$ and kidneys $(+)$ in the propositus Case A III-3. The SAP scan in both his sisters Case A III-4 and Case A III-5 showed 
only a positive spleen, whereas no abnormal uptake was seen in Case A IV-1.

The SAP scan in Case B showed a moderate total body amyloid with abnormal splenic uptake and an equivocal signal from the kidneys.

\section{TTR mutation}

The TTR gene in patients and family members was amplified and analysed as previously described ${ }^{11}$ using an AB1310 sequencing machine and Big Dye (PE Applied Biocystems, Cheshire, UK) sequencing chemistry. See Figure 1.

\section{Discussion}

Characterisation of the clinical features and natural history of disease associated with rare amyloidogenic TTR mutations offers the best guidance for management of individual patients, in particular with regard to the possible role and timing of treatment by orthotopic liver transplantation. Although FAP associated with TTR Gly47Glu has only been identified in a few families in total, it is evidently present in diverse populations, and is very unusual in that renal involvement is a major clinical feature. The clinical features associated with TTR Gly47Glu in the patients reported here were in other respects less remarkable, but notable features included relatively mild peripheral neuropathy in most cases, and frequently severe autonomic and cardiac involvement. The age of onset varied from 33 to 69 years, and late onset or absence of apparent disease had obscured the family history in several of our cases. Vitreous amyloid deposits were not present in any of the patients reported here.

The only previous description of ATTR Gly47Glu has been in a single Italian family with FAP, two members of which were described in detail ${ }^{6}$. This report suggests that peripheral neuropathy may have been an earlier and more predominant feature, but autonomic dysfunction, amyloid cardiomyopathy and renal involvement were similarly present. Indeed, the severity of cardiac and renal involvement in the Italian kindred is emphasised by the frequent development of clinically significant cardiac failure and need for hemodialysis in some members, the latter being exceptionally rare in the natural history of FAP.

The prominent nature of visceral amyloid deposition in ATTR Gly47Glu was supported by the findings of SAP scintigraphy in four of the five patients reported here who underwent this investigation. Amyloid deposits in the spleen were imaged in four patients and renal deposits in two cases, the latter reflecting the degree of clinical renal involvement. In line with previous experience, SAP scintigraphy was unable to image cardiac amyloid deposits. This may be caused by the high molecular weight of SAP which hampers access to amyloid deposits in cardiac tissue. In this respect, the development of tracers with low molecular weight, such as DPD, might have additional value in the evaluation of cardiac disease ${ }^{12}$. The findings of SAP scintigraphy in FAP have shown reasonably consistency between the degree of visceral amyloid involvement associated with different TTR mutations. For example, almost all patients with ATTR Val30Met show minor deposits in the kidneys and/or spleen, whereas these images are normal in virtually all patients with ATTR Thr60Ala, Ser77Tyr and Val122Ile, which represent some of the most common variants. SAP scintigraphy has shown more substantial visceral involvement in patients with other TTR variants of FAP that are associated with a severe clinical phenotype, including TTR Leu12Pro ${ }^{13}$, and Ser52Pro in two British families. SAP scintigraphy therefore contributes to the characterisation of FAP in individual patients and in studies of particular mutations, as well as having a unique role in serially monitoring visceral amyloid deposits in FAP following liver transplantation. Such follow-up studies have so far shown stabilisation or regression of amyloid deposits in the kidneys, spleen and adrenal glands after liver transplantation in all patients with FAP regardless of mutation ${ }^{14}$. This is reassuring in light of the reports of progressive cardiomyopathy in this situation, which can be rapid and severe.

Orthotopic liver transplantation remains the only treatment that is presently available for FAP associated with genetic variants of TTR, but it is not viable in patients who have significant cardiac amyloidosis or in those with renal failure. Combined heart and liver transplantation is feasible in some FAP patients with severe cardiac amyloidosis, but such treatment is hazardous and is rarely performed in practice. Liver and kidney transplantations have been performed together with success in FAP, but simultaneous liver, heart and renal grafting has not yet been attempted. The wider experience of liver transplantation in FAP indicates that early intervention is associated with the most favourable outcomes. Indeed, because both cardiac and renal amyloidosis can be severe in FAP without causing symptoms, and yet preclude isolated liver transplantation, there may be a case to proceed to liver transplantation before the development of any symptoms at all in individuals who have been identified to have ATTR Gly47Glu and who have well documented sub-clinical amyloid disease. On the other hand, long term benefit of 'pre-emptive' liver transplantation has not yet been 
demonstrated and, for example, it is a possibility that progressive amyloid cardiomyopathy might still occur even if only microscopic myocardial amyloid deposits were present at the time of surgery.

In conclusion, our findings confirm that ATTR Gly47Glu appears to be dominated by early and progressive involvement of the cardiovascular system, and often the kidneys. Isolated liver transplantation is therefore only likely to be feasible at a very early stage in most cases.

\section{Acknowledgments}

We would like to thank Johan Bijzet for his technical assistance and Piet L. Jager for performing the SAP scintigraphs of the Dutch cases.

\section{References}

1 Benson MD and Uemichi T (1996). Tranthyretin amyloidosis. Amyloid: Int J Exp Clin Invest 3, 44-56

2 Ericzon BG, Holmgren G, Lundgren E and Suhr OB (2000). New structural information and update on liver transplantation in transthyretin associated amyloidosis. Report from the 4th International Symposium on Familial Amyloidotic Polyneuropathy and Other Transthyretin Related Disorders and the 3rd International Workshop on Liver Transplantation in Familial Amyloid Polyneuropathy, Umea, Sweden, June 1999. Amyloid Int J Exp Clin Invest 7, 145-147

3 Dubrey SW, Davidoff R, Skinner M, Bergethon P, Lewis D and Falk RH (1997). Progression of ventricular wall thickening after liver transplantation for familial amyloidosis. Transplantation $\mathbf{6 4}, \mathbf{7 4 - 8 0}$

4 Olofsson BO, Backman C, Karp K and Suhr OB (2002). Progression of cardiomyopathy after liver transplantation in patients with familial amyloidotic polyneuropathy, Portuguese type. Transplantation 73, 745-751

5 Altland K (1999). Common molecular characteristics of amyloidogenic TTR mutations. In: Program and Abstracts from The 4th International Symposium on Familial Amyloidotic Polyneuropathy and Other Transthyretin Related Disorders \& The 3rd International Workshop on Liver Transplantation in Familial Amyloid Polyneuropathy, Umeå, Sweden, June 3-5, 1999. Abstract, page 17
6 Pelo E, Da Prato L, Ciaccheri M, Castelli G, Gori F, Pizzi A, Torricelli F and Marconi (2002). Familial amyloid polyneuropathy with genetic anticipation associated to a gly47glu transthyretin variant in an Italian kindred. Amyloid: J. Protein Folding Disord. 9, 35-41

7 Lachmann MB, Booth DR, Booth SE, Bybee A, Gilbertson JA, Gillmore JD, Pepys MB and Hawkins PN (2002). Misdiagnosis of hereditary amyloidosis as AL (primary) amyloidosis. $N$ Engl J Med 346, 1786-1791

8 Mather SJ and Ward BC (1987). High efficiency iodination of monoclonal antibodies for radiotherapy. J Nucl Med 28, 1034-1036

9 Jager PL, Hazenberg BPC, Franssen EJF, Limburg PC, Van Rijswijk MH and Piers DA (1998). Kinetic studies with iodine-123-labelled serum amyloid $\mathrm{P}$ component in patients with systemic AA and AL amyloidosis and assessment of clinical value. J Nucl Med 39, 699-706

10 Hawkins PN, Lavender JP and Pepys MB (1990). Evaluation of systemic amyloidosis by scintigraphy with 123I-labelled serum amyloid P component. $N$ Engl J Med 323, 508-513

11 Booth DR, Tan SY, Hawkins PN, Pepys MB and Frustaci A (1995). A novel variant of transthyretin, 59 $9^{\text {Thr-Lys, }}$ associated with autosomal dominant cardiac amyloidosis in an Italian family. Circulation 91, 962-967

12 Puille M, Altland K, Linke RP, Steen-Muller MK, Kiett R, Steiner D and Bauer R (2002). 99mTc-DPD scintigraphy in transthyretin-related familial amyloidotic polyneuropathy. Eur J Nucl Med Mol Imaging 29, 376-379

13 Brett M, Persey MR, Reilly MM, Revesz T, Booth DR, Booth SE, Hawkins PN, Pepys MB and Morgan-Hughes JA (1999). Transthyretin Leu12Pro is associated with systemic, neuropathic and leptomeningeal amyloidosis. Brain 122, 183-190

14 Rydh A, Suhr O, Hietala S-O, Åhlström KR, Pepys MB and Hawkins PN (1998). Serum amyloid P component scintigraphy in familial amyloid polyneuropathy: regression of visceral amyloid following liver transplantation. Eur J Nucl Med 25: 709-713 